\title{
Crystal-Face Dependence of Surface Melting
}

\author{
B. Pluis, A. W. Denier van der Gon, J. W. M. Frenken, ${ }^{(a)}$ and J. F. van der Veen \\ FOM-Institute for Atomic and Molecular Physics, 1098 SJ Amsterdam, The Netherlands
} (Received 14 April 1987)

\begin{abstract}
Ion-shadowing and -blocking experiments on a cylindrical single crystal of $\mathrm{Pb}$ reveal a strongly orientation-dependent disordering (melting) of the surface with increasing temperature. The thickness of the disordered surface layer is found to diverge logarithmically as the bulk melting point is approached. The process of disordering is shown to be driven by an orientation-dependent difference in free energy between the surface in its ordered and liquid states.
\end{abstract}

PACS numbers: $68.35 . \mathrm{Rh}, 68.35 . \mathrm{Md}, 68.45 . \mathrm{Gd}$

The possible role of the surface in initiating the melting of a solid has long been debated. ${ }^{\prime}$ A recent observation of surface melting on an atomic level ${ }^{2}$ has created a surge of renewed interest in the phenomenon. ${ }^{3-6}$ Our present state of knowledge can be summarized as follows. Surface melting is an intrinsic effect. ${ }^{7}$ It involves a positional disordering of the lattice in the surface region just below the bulk melting point and is to be distinguished from "surface roughening." As the temperature $T$ approaches the bulk melting point $T_{m}$, the melted layer thickness $d$ is predicted to diverge as $\left|\ln \left(T_{m}-T\right)\right|$ or as $\left(T_{m}-T\right)^{-r}$, depending on whether the acting forces are of short or long range. ${ }^{8,9}$ The melted layer is not a true liquid, since the underlying lattice induces some crystalline order in it. ${ }^{10}$ Hence it is usually referred to as a "quasiliquid."

Several predictions have been made regarding a variation in melting behavior with surface orientation. ${ }^{3,11}$ Crystal faces with open packing of atoms are expected to melt readily, whereas close-packed faces may not melt at all below $T_{m}$. Such a trend has been observed by Stock $^{12}$ in optical emission measurements on spherical $\mathrm{Cu}$ monocrystals which were heated up to $T_{m}$.

In this Letter we report the first measurements of melted-layer thickness as a function of surface orientation and temperature. With use of medium-energy ion scattering, the presence of melting-related disorder was detected in the outermost atomic layers of a $\mathrm{Pb}$ crystal which was cylindrically cut so as to expose a range of surface orientations. The number of disordered monolayers at a given temperature in the vicinity of $T_{m}$ was found to vary dramatically with surface orientation. A simple thermodynamic model quantitatively explains this result.

A cylindrical specimen was spark cut from a singlecrystal $\mathrm{Pb}$ bar of high purity. ${ }^{13}$ The cylinder surface exposes a $73^{\circ}$ range of crystallographic orientations along the $[\overline{1} 10]$ zone of the stereographic triangle. The orientation of a particular face on the cylinder is defined by the angle $\theta$ between its normal and the [112] axis (Fig. 1). The surface was prepared as in Ref. 7. It was found to be well ordered as seen with low-energy electron dif- fraction and ion channeling. With Auger-electron spectroscopy it was checked that the surface was atomically clean. No segregation of impurities was observed with increasing $T$. The surface temperature was monitored by a pyrometer which was calibrated against a $\mathrm{Pt}$ resistance. The temperature scale was fixed by the bulk melting point of $\mathrm{Pb}$, which was measured in situ. Close to $T_{m}$, the temperature was determined with an absolute accuracy of $\pm 0.1 \mathrm{~K},{ }^{7}$ and the temperature stability and uniformity over the surface was found to be within $0.05 \mathrm{~K}$.

The scattering geometry is shown in Fig. 1 for a wellordered crystal. A collimated beam of $75.6-\mathrm{keV} \mathrm{H}^{+}$was aligned with the [1 $10 \overline{1}]$ crystal axis and only those backscattered protons were detected which emerged from the crystal in a direction parallel to the [121] axis. The shadowing and blocking effects which arise in this geometry along the [1]01] and [121] atomic rows, respectively, cause the contribution from subsurface atoms to the

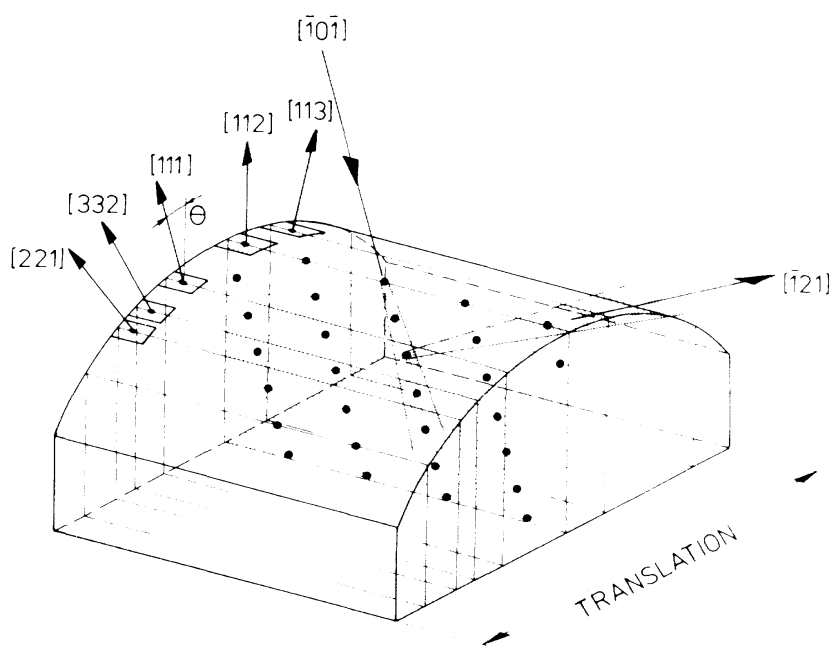

FIG. 1. Ion shadowing and blocking geometry in the (11i) scattering plane of a cylindrically shaped $\mathrm{Pb}$ crystal. The cylinder surface exposes a $73^{\circ}$ range of crystallographic orientations along the $[\overline{1} 10]$ zone of the stereographic triangle. 
backscattered yield to decrease rapidly with depth. ${ }^{2,7}$ The energy spectrum of backscattered protons therefore exhibits a distinct "surface peak." With use of established calibration procedures, the measured surface-peak area is expressed as the effective number $L$ of "visible" (i.e., nonshadowed and nonblocked) atoms per [ $\overline{1} 0 \overline{1}]$ row.

An increased (anharmonic) thermal vibration amplitude of the atoms along the atomic rows reduces the shadowing and blocking effects and causes $L$ to increase with $T .{ }^{14}$ In case the surface melts, the disorderly positioned atoms in the melted region no longer contribute coherently to shadowing or blocking along the rows, and $L$ rises additionally. ${ }^{2,7}$

The crystal-face dependence of surface melting was investigated by our translating the crystal, at fixed $T$, in discrete steps (Fig. 1) and measuring at each setting the number $L(T, \theta)$ of visible atoms per row for the corresponding orientation angle $\theta$. The alignment of beam and detector with respect to $[\overline{1} 0 \overline{1}]$ and $[\overline{1} 21]$ crystal axes is preserved in this procedure. Hence, for a well-ordered surface, $L$ essentially remains unchanged upon translation (apart from minor variations related to an orientation dependence in the surface relaxation and the surface vibration amplitude). For a melted surface, however, $L$ will be higher. Any significant change in $L$ with translation setting must be the consequence of an orientationdependent variation in the degree of disorder.

The measured $T$ dependence of the surface-peak area is shown in Fig. 2 for some selected orientations. The number of atoms per row $L$ for the (332) and (221) faces first follows the curve for the (111) face up to $\simeq 500 \mathrm{~K}$, but then rises dramatically as $T$ approaches

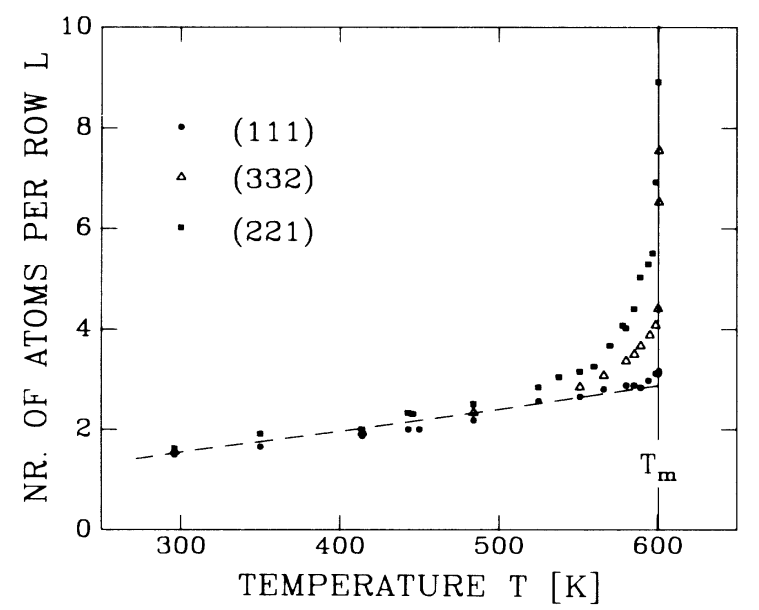

FIG. 2. Number of visible atoms per row $L$ measured for the (111), (332), and (221) faces of $\mathrm{Pb}$, as a function of temperature. The vertical line indicates the bulk melting point $T_{m}$ at $600.7 \mathrm{~K}$. The dashed curve is the result of a Monte Carlo simulation for a well-ordered surface with thermally vibrating atoms on truncated-bulk positions.
$T_{m}$. A similar rapid increase of the disorder has been reported earlier for the $\mathrm{Pb}(110)$ surface and has been shown to be a direct manifestation of surface melting. ${ }^{2,7}$ Clearly, the (332) and (221) faces melt earlier than the bulk, whereas the close-packed (111) face does not.

The absence of any premelting effect on the (111) surface is deduced from the fact that the measurements are in excellent agreement, up to $T_{m}$, with Monte Carlo computer simulations ${ }^{14}$ of the experiment (dashed curve in Fig. 2). In these simulations the surface was assumed to be well ordered at all temperatures. The lattice vibrations were modeled by Gaussian probability densities for the displacements of the atoms around their equilibrium positions. The one-dimensional root mean square thermal displacement of the atoms in the bulk was raised from $0.18 \AA$ at $300 \mathrm{~K}$ to $0.28 \AA$ just below $T_{m}{ }^{7}$ The vibration amplitude of the surface atoms was assumed to be enhanced by $15 \%$ with respect to the bulk value and the surface structure was taken to be a truncation of the bulk lattice (i.e., no relaxation).

Rather than $L(T, \theta)$ the parameter of interest is the number $N(T, \theta)$ of disorderly positioned atoms per unit area, which follows from the relation $N(T, \theta)$ $=N_{s}\left[L(T, \theta)-L_{\text {ord }}(T)\right] \cos \theta$. Here, $N_{S}$ is the areal density of atomic rows terminating the (112) crystal face $\left(N_{S}=1.000 \times 10^{15} \mathrm{~cm}^{-2}\right)$, and $L_{\text {ord }}(T)$ is the number of atoms per row calculated for an ordered surface by Monte Carlo simulation. The $\theta$ dependence of $N(T, \theta)$ is shown in Fig. 3 for various temperatures close to $T_{m}$. Striking are the absence of positional disorder in $\mathrm{a} \simeq 17^{\circ}$

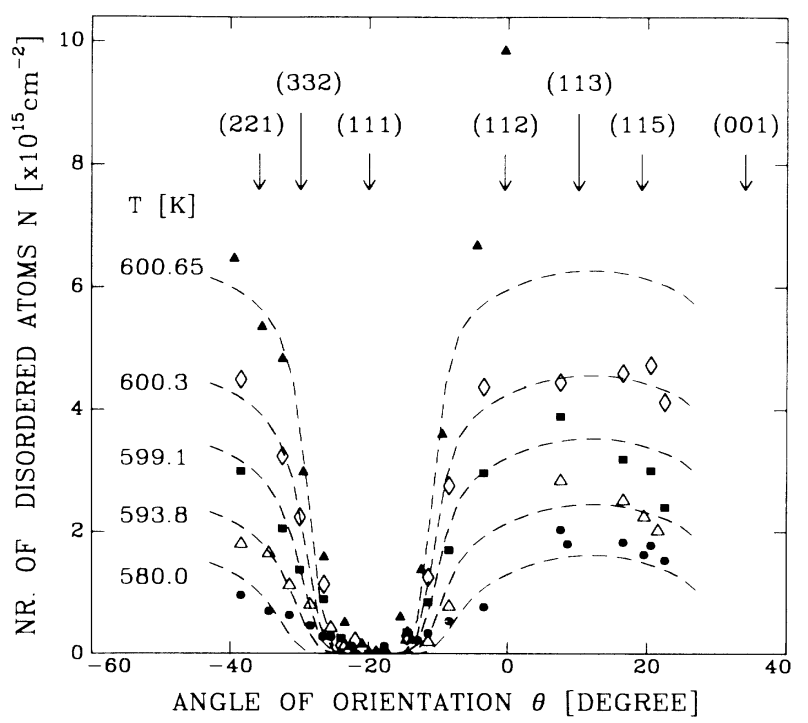

FIG. 3. Number of disordered atoms per unit area $N$ as a function of surface orientation angle $\theta$ with respect to the [112] axis, measured at various temperatures starting from $=200 \mathrm{~K}$ below the bulk melting point $T_{m}=600.7 \mathrm{~K}$. The dashed curves represent the optimal fit of Eq. (2) to the data (see text). 
wide zone around the (111) orientation and the rapid rise of $N$ at either side of this zone. $N$ decreases again as the $(001)$ orientation is approached, suggesting that the (001) face does not melt either.

The data are strongly suggestive of a correlation with the free energy $\gamma_{\mathrm{sv}}(\theta)$ of the ordered-solid-vapor interface, which is known to be orientation dependent (Fig. 4) ${ }^{15}$; the angles at which extrema occur in $\gamma_{\mathrm{sv}}(\theta)$ and $N(T, \theta)$, coincide. An explicit relationship between $\gamma_{\mathrm{sv}}(\theta)$ and $N(T, \theta)$ will first be given and then tested in a comparison with the data.

Consider a quasiliquid layer to be present at the boundary of solid and vapor. The total free energy per unit area of the boundary is then given by ${ }^{8}$

$$
\begin{array}{rl}
\gamma=\gamma_{\mathrm{sl}}+\gamma_{\mathrm{lv}}+\mathcal{L} & \mathcal{L}\left(1-T / T_{m}\right) \\
+ & \left(\gamma_{\mathrm{sv}}-\gamma_{\mathrm{sl}}-\gamma_{\mathrm{lv}}\right) \exp \left(-N / N_{0}\right),
\end{array}
$$

where $\gamma_{\mathrm{s}}$ and $\gamma_{\mathrm{lv}}$ are the free energies per unit area of the solid-liquid (sl) and liquid-vapor (lv) interfaces, $\mathcal{L}$ the latent heat of melting per atom, $N_{0}$ a constant, and $N$ the number of atoms per unit area in the quasiliquid layer. $N$ relates to the layer thickness $d$ through $N=n d$, where $n$ is the atomic concentration (for $\mathrm{Pb}, n=3.30$ $\times 10^{22} \mathrm{~cm}^{-3}$ ). The third term in Eq. (1) represents the free energy associated with undercooling of the quasiliquid layer. The fourth term corrects for the layer's not being a true liquid but a quasiliquid with an effective crystalline order parameter $\exp \left(-N / N_{0}\right)$. This choice of order parameter is appropriate for a system governed by short-range forces. ${ }^{8}$ Minimizing $\gamma$ with respect to $N$ yields the orientation-dependent number of atoms $N_{\text {eq }}$ in the quasiliquid layer at equilibrium:

$$
N_{\mathrm{eq}}(T, \theta)=N_{0} \ln \left\{\frac{T_{m} \Delta \gamma(\theta)}{\left(T_{m}-T\right) \mathcal{L} N_{0}}\right\},
$$

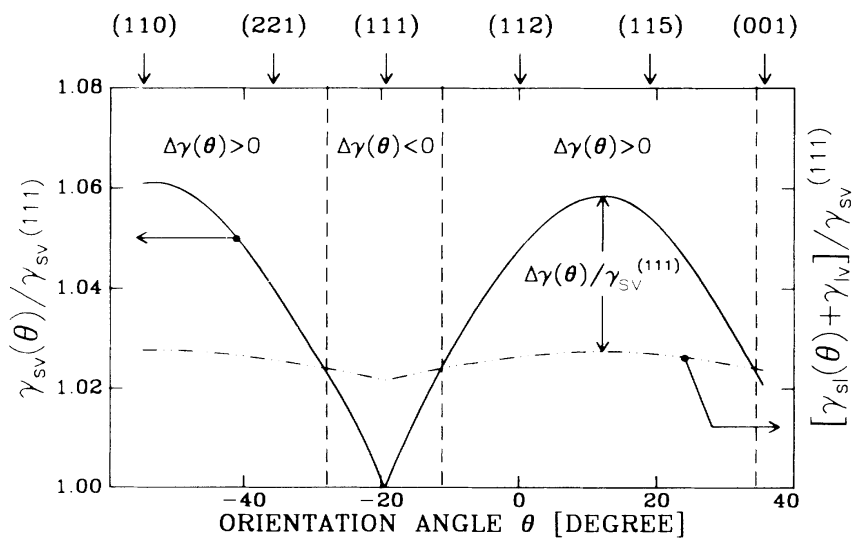

FIG. 4. Normalized free energy of the $\mathrm{Pb}$ solid-vapor interface $\gamma_{\mathrm{sv}}(\theta) / \gamma_{\mathrm{sv}}^{(111)}$, as measured by Heyraud and Métois (Ref. 15) at $T=473 \mathrm{~K}$ (solid curve). The dash-dotted curve represents the normalized sum $\left[\gamma_{\mathrm{sl}}(\theta)+\gamma_{\mathrm{lv}}\right] / \gamma_{\mathrm{sv}}^{(111)}$, obtained by fitting of Eq. (2) to the data of Fig. 3 (see text). where $\Delta \gamma(\theta)=\gamma_{\mathrm{sv}}(\theta)-\gamma_{\mathrm{sl}}(\theta)-\gamma_{\mathrm{lv}}$ is the free energy which an ordered solid surface has in excess of a surface with a liquid layer on top. Clearly, the parameter controlling the angular dependence of $N_{\text {eq }}(T, \theta)$ is the anisotropic excess free energy $\Delta \gamma(\theta)$. Surface melting will only occur on crystal faces for which $\Delta \gamma(\theta)>0$.

The above model predicts values for $N_{\text {eq }}(T, \theta)$ at temperatures close to $T_{m}$ which are in quantitative agreement with the experimental data. The most important input parameter of the model is $\gamma_{\mathrm{sv}}(\theta)=\left[\gamma_{\mathrm{sv}}(\theta) /\right.$ $\left.\gamma_{\mathrm{sv}}^{(11)}\right] \gamma_{\mathrm{sv}}^{(11)}$. The ratio $\gamma_{\mathrm{sv}}(\theta) / \gamma_{\mathrm{sv}}^{(111)}$ describing the anisotropy is taken from the work of Heyraud and Métois ${ }^{15}$ (Fig. 4) and for $\gamma_{\mathrm{sv}}^{(111)}$ the value of $0.544 \mathrm{~J} / \mathrm{m}^{2}$ is taken. ${ }^{16}$ The solid-liquid interface energy $\gamma_{\mathrm{sl}}(\theta)$ is obtained with the empirical rule ${ }^{17} \gamma_{\mathrm{sl}}(\theta)=0.1 \gamma_{\mathrm{sv}}(\theta)$. The two remaining unknowns in Eq. (2), $\gamma_{l v}$ and $N_{0}$, are treated as free parameters to be determined from a fit to the data in Fig. 3. To allow for a proper comparison with experiment, the calculated angle dependence of $N_{\mathrm{eq}}(T, \theta)$ is convoluted with a Gaussian spread in $\theta$ having a full width at half maximum of $3.6^{\circ}$. This takes into account the fact that the proton beam samples $a=3.6^{\circ}$ range of orientations over the cylinder surface. A good fit to all data in Fig. 3 is obtained for $\gamma_{l v}=0.501 \mathrm{~J} / \mathrm{m}^{2}$ and $N_{0}=7.32 \times 10^{14} \mathrm{~cm}^{-2}$ (dashed lines in Fig. 3). ${ }^{18}$ The best-fit value for $\gamma_{l v}$ is close to the average value of 0.46 $\mathrm{J} / \mathrm{m}^{2}$ known from the literature. ${ }^{19}$ The best-fit value of $N_{0}$ is smaller than the value of $20.56 \times 10^{14} \mathrm{~cm}^{-2}(6.23$ $\AA$ ) found previously for $\mathrm{Pb}(110) .^{7}$ The difference is probably related to a crystal-face-dependent variation of the crystalline order profile across the solid-quasiliquid interface. The latter issue is currently being explored.

A graphical representation of our melting model for $\mathrm{Pb}$ is shown in Fig. 4. In this figure the normalized sum

$$
\left[\gamma_{\mathrm{sl}}(\theta)+\gamma_{\mathrm{lv}}\right] / \gamma_{\mathrm{sv}}^{(111)}=0.10 \gamma_{\mathrm{sv}}(\theta) / \gamma_{\mathrm{sv}}^{(111)}+0.501 / 0.544
$$

(dash-dotted curve) is compared with $\gamma_{\mathrm{sv}}(\theta) / \gamma_{\mathrm{sv}}^{(111)}$ (solid curve) over the full range of orientation angles for which the latter quantity is known. ${ }^{15}$ The angles $\theta$ at which the curves intersect define the boundaries of the nonmelted zones (dashed vertical lines). Around the (111) orientation the zone is $17^{\circ}$ wide, as is experimentally observed (Fig. 3). For (001) no melting data are available, but the model predicts this crystal face to be on the verge of melting.

In conclusion, the atomic-scale melting of a surface is driven by the excess free energy $\Delta \gamma(\theta)=\gamma_{\mathrm{sv}}(\theta)-\gamma_{\mathrm{sl}}(\theta)$ $-\gamma_{\mathrm{lv}}$. For $\mathrm{Pb}$, the condition for surface melting is met for many crystal faces, but not for a well-defined zone around the (111) orientation and, possibly, a limited zone around (001). It needs to be explored whether materials other than $\mathrm{Pb}$ also exhibit melting crystal faces. If this is generally the case, then we have identified a microscopic phenomenon of important macroscopic consequences. The surface of a solid of finite dimensions will then always contain regions where the melting condition 
$\Delta \gamma(\theta)>0$ is valid. Hence, melting of a solid generally commences at the surface. Of course, impurities, oxides, etc., which may be present on a "practical" surface may modify $\Delta \gamma(\theta)$, thereby promoting or suppressing surface-initiated melting. The latter effect is presently under investigation.

A. J. Riemersma and B. Moleman from the University of Amsterdam are gratefully acknowledged for their careful preparation of our $\mathrm{Pb}$ specimens. We thank $\mathrm{Dr}$. K. C. Prince from Kernforschungsanlage Jülich $\mathrm{GmbH}$ for having provided us with a detailed trace-element analysis of the $\mathrm{Pb}$ crystal bar. This work is sponsored by the Stichting voor Fundamenteel Onderzoek der Materie (FOM) with financial support from the Nederlandse Organisatie voor Zuiver-Wetenschappelijk Onderzoek (ZWO).

(a) Present address: Max-Planck-Institut für Strömungsforschung, Bunsenstrasse 10, 3400 Göttingen, Federal Republic of Germany.

${ }^{1}$ G. Tammann, Z. Phys. Chem. 68, 205 (1910).

2J. W. M. Frenken and J. F. van der Veen, Phys. Rev. Lett. 54, 134 (1985).

${ }^{3}$ J. Q. Broughton and G. H. Gilmer, Phys. Rev. Lett. 56, 2692 (1986).

${ }^{4}$ T. Nguyen, P. S. Ho, T. Kwok, C. Nitta, and S. Yip, Phys. Rev. Lett. 57, 1919 (1986).
${ }^{5}$ G. Devaud and R. H. Willens, Phys. Rev. Lett. 57, 2683 (1986).

${ }^{6}$ Da-Ming Zhu and J. G. Dash, Phys. Rev. Lett. 57, 2959 (1986).

${ }^{7}$ J. W. M. Frenken, P. M. J. Marée, and J. F. van der Veen, Phys. Rev. B 34, 7506 (1986).

${ }^{8}$ J. Q. Broughton and G. H. Gilmer, Acta Metall. 31, 845 (1983).

${ }^{9}$ J. K. Kristensen and R. M. J. Cotterill, Philos. Mag. 36, 437 (1977).

${ }^{10}$ R. Lipowsky and W. Speth, Phys. Rev. B 28, 3983 (1983).

${ }^{11}$ C. S. Jayanthi, E. Tosatti, and L. Pietronero, Phys. Rev. B 31, 3456 (1985).

${ }^{12}$ K. D. Stock, Surf. Sci. 91, 655 (1980).

${ }^{13}$ It was verified by spark mass spectrometry that the bulk concentrations of melting-point-lowering impurity elements such as $\mathrm{Bi}, \mathrm{Ag}$, etc., were below the $=5 \mathrm{ppm}$ level (K. C. Prince, private communication).

${ }^{14}$ J. W. M. Frenken, R. M. Tromp, and J. F. van der Veen, Nucl. Instrum. Methods. Phys. Res., Sect. B 17, 334 (1986).

${ }^{15}$ J. C. Heyraud and J. J. Métois, Surf. Sci. 128, 334 (1983).

${ }^{16}$ A. R. Miedema, Z. Metallkd. 69, 287 (1978).

${ }^{17}$ A. R. Miedema and F. J. A. den Broeder, Z. Metallkd. 70, 14 (1979), and references therein.

${ }^{18}$ Note that some of the $N(T, \theta)$ values, measured at $T=600.65 \mathrm{~K}$, deviate from the fit. This is probably an artifact caused by a small temperature gradient of $\simeq 0.02 \mathrm{~K}$ over the sample surface.

${ }^{19}$ A. R. Miedema and R. Boom, Z. Metallkd. 69, 183 (1978), and references therein. 